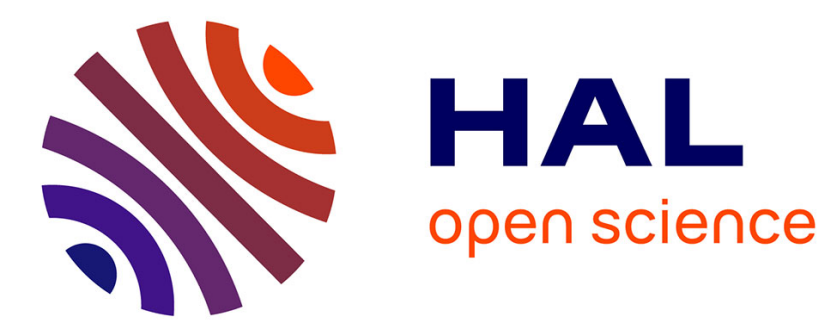

\title{
Scoring Rules and Preference Restrictions: The Strong Borda Paradox Revisited
}

\author{
Eric Kamwa, Fabrice Valognes
}

\section{To cite this version:}

Eric Kamwa, Fabrice Valognes. Scoring Rules and Preference Restrictions: The Strong Borda Paradox Revisited. Revue d'Economie Politique, 2017, 127 (3), pp.375-395. 10.3917/redp.273.0375 . hal01631180

\section{HAL Id: hal-01631180 \\ https://hal.univ-antilles.fr/hal-01631180}

Submitted on 27 Jun 2019

HAL is a multi-disciplinary open access archive for the deposit and dissemination of scientific research documents, whether they are published or not. The documents may come from teaching and research institutions in France or abroad, or from public or private research centers.
L'archive ouverte pluridisciplinaire $\mathbf{H A L}$, est destinée au dépôt et à la diffusion de documents scientifiques de niveau recherche, publiés ou non, émanant des établissements d'enseignement et de recherche français ou étrangers, des laboratoires publics ou privés. 


\title{
Scoring rules and preference restrictions: The Strong Borda Paradox revisited*
}

\author{
Eric Kamwa $\quad$ Fabrice Valognes ${ }^{\ddagger}$
}

November 16, 2016

\begin{abstract}
For a given voting situation, the Strong Borda Paradox occurs when a Condorcet loser exists and is elected. A Condorcet loser is a candidate that loses all his pairwise comparisons. In three-candidate elections, we use an analytical approach to find out the range of all the scoring rules that can exhibit the Strong Borda Paradox under some well-known preference restrictions and we describe all the scenarios with respect to the rank of the Condorcet loser in the collective rankings. Using the parameterized Barvinok's algorithm, we provide a simplified representation of the likelihood of the Strong Borda Paradox for the Plurality rule and the Antiplurality rule (given the size of the electorate) with the impartial and anonymous culture condition for each type of restriction.
\end{abstract}

Keyword: Scoring rules - Condorcet - Preference restrictions - Strong Borda Paradox - Impartial Anonymous Culture.

${ }^{*}$ The authors are grateful to Bill Gehrlein for his useful remarks. They also thank an anonymous referee for his valuable comments.

${ }^{\dagger}$ Université des Antilles. Faculté de Droit et d'Economie de la Martinique, Campus de Schoelcher BP7209, F97209 Schoelcher, Martinique. (凶):eric.kamwa@univ-antilles.fr, Corresponding author.

${ }^{\ddagger}$ Normandie Univ, France ; UNICAEN, CREM, UMR-CNRS 6211. UFR Sciences Econ, Gestion, Géographie et Aménagement du Territoire, Esplanade de la Paix, F14032 Caen. fabrice.valognes@unicaen.fr 


\section{Règles de scores et restrictions de préférences: le Paradoxe Fort de Borda revisité}

\section{Résumé}

Pour une situation de vote, le paradoxe fort de Borda se produit lorsqu'il existe un perdant de Condorcet et que ce dernier est élu. Un perdant de Condorcet est un candidat battu dans chacun de ses duels majoritaires. Pour des situations de vote avec trois candidats, nous utilisons une approche analytique pour déterminer l'ensemble de toutes les règles de scores vulnérables au paradoxe fort de Borda ceci sous certaines restrictions préférences; aussi, nous décrivons tous les scénarios concernant le rang du perdant de Condorcet dans le classement collectif. A partir de l'algorithme paramétré de Barvinok et sous l'hypothèse de culture neutre et anonyme, nous proposons pour chaque type de restriction des préférences, une représentation simplifiée des probabilités du paradoxe fort de Borda pour les règles de la Pluralité simple et de l'Antipluralité.

Mots-clés: Règles de score - Condorcet - Restriction des préférences - Paradoxe fort de Borda - Culture neutre et anonyme.

JEL Classification: D70, D71

\section{Introduction}

Majority cycles are troublesome things that can occur under preferences aggregation. Nonetheless, they could be avoided if some restrictions are made over the preferences (Black, 1958). With restrictions, voters are only allowed to have some particular or predefined types of preferences over the set of alternatives (candidates). Therefore, the choice set is no longer the universal domain but a restricted one. In the social choice literature, there are three well-known restrictions that prevent cycles. The most known is the Black's single-peakedness (Black, 1958) also called the Never Bottom-Ranked restriction (NBR) (see Saari and Valognes, 1999). The NBR requires that there are some candidates who are never ranked last by the voters. The Never Middle-Ranked restriction (NMR) (Ward, 1965) requires that there are some candidates never middle-ranked by the voters ${ }^{1}$. The Never Top-Ranked restriction (NTR) (Vickery, 1960) imposes that there are some candidates that no voter ranks

\footnotetext{
${ }^{1}$ Preferences are also said to be separable (Sen, 1966).
} 
at the top of her ranking ${ }^{2}$. These restrictions can be used to define measures of homogeneity of preferences which is the proximity of a voting situation to meet the condition of single-peakedness with the NBR, of single-dippedness with the NTR, of perfect polarization with the $\mathrm{NMR}^{3}$.

The NBR describes situations where it is appropriate to represent policy options on a one-dimensional axis such as the ideological positions or the possible values of a tax rate on the Left-Right axis. On this axis, a voter will be inclined to vote for an option if it is closer to his preferred position (his bliss point). The NBR is used in one of the main theoretical results that underpin much of the analysis of the new Political Economy: the median voter theorem (Black, 1958). According to Gehrlein (2004), a never bottom-ranked candidate appears as a positively unifying candidate since no voter is against her possible election.

The never top-ranked candidate is a negatively unifying candidate since none of the voters think that his election is the best possible outcome. The NTR naturally arises in the presence of a public bad (a facility whose neighborhood is undesirable). For example, assume that voters have to decide on where to locate three projects: a garden, a nuclear plant and a stadium; it is natural to think that for each voter, the locations become better as the worse project (here, the nuclear plant) is further away of his home. So, as to locate the good close to their houses, no voter ranks the nuclear plant at the top.

Notice that even if the NBR and the NTR refer to a form of consistency of preferences, this is less obvious for the NMR which is characterized by the existence of a cleaving option (a perfectly polarizing candidate). A good illustration of a cleaving option can be drawn from the U.S political arena where Republicans and Democrats are opposed. Assume that voters have to decide on three bills to enact: one on renewable energies, one on lowering taxes and one on security and armament. It is clear that the bill on renewable energies will appear as the cleaving option since Republicans are "climate skeptics" ${ }^{4}$ " while the Democrats will more promote ecology than armament and taxes lowering.

Thereby, the NBR, the NMR and the NTR do not only have a theoretical scope but they help in explaining how people may behave in certain situations. Although

\footnotetext{
${ }^{2}$ With three candidates, preferences are also said to be Single-Dipped (Sen, 1966). Sen (1966) showed that with three candidates, if preferences are either Single-peaked, Single-Dipped or Separable, there always exists a Condorcet winner. A Condorcet winner is a candidate that beats each of the other candidates in pairwise contests.

${ }^{3}$ We refer to Gehrlein and Lepelley (2010a) for more on this.

${ }^{4}$ During the 2016 Republican presidential primaries, all the candidates agreed to go back on the commitments made by Obama regarding the reduction of greenhouse gases, even those taken during the COP21.
} 
these restrictions are helpful in avoiding cycles, Saari and Valognes (1999) used an example to show that unfortunately, for some voting rules, they do not prevent the election of a candidate that loses all his pairwise comparisons viz. the Condorcet loser. Notice that under our restrictions, the Condorcet loser always exists at least for an odd number of voters; for an even number of voters, the probability of a tie quickly becomes close to 0 as the size of the electorate grows. Almost all the social choice theorists agree that the election of such a candidate when she exists is an unacceptable voting outcome. A voting rule that can elect a Condorcet loser is said to be vulnerable to the Strong Borda Paradox (SgBP). Among the scoring rules ${ }^{5}$, it is known that when no restriction is made on the preferences, only the Borda rule ${ }^{6}$ is immune to the $\mathrm{SgBP}^{7}$.

In three-candidate elections, when the NBR is assumed, Lepelley (1993) showed that the Antiplurality rule ${ }^{8}$ never elects the Condorcet loser. According to Lepelley (1996), under the NBR there may exist other scoring rules immune to the SgBP under certain conditions (see Lepelley (1996, Theorem 1)). When we restrict the framework of Lepelley (1996) to voting situations with three candidates, only the Borda rule and the Antiplurality rule are immune to the SgBP (see also Gehrlein and Lepelley (2010a)). For three-candidate elections, Lepelley et al. (2000b), Gehrlein and Lepelley (2010a) provided a representation of the limiting probability (with an infinite number of voters) of the SgBP for all the scoring rules given the NBR. They concluded that: in three-candidate elections, the Strong Borda Paradox never occurs for all the scoring rules located between the Borda rule and the Antiplurality rule. So, with three candidates, the Plurality rule is one of the scoring rules that are vulnerable to the SgBP when preferences are restricted according to the NBR. Under the NBR, Lepelley (1993) has computed the likelihood of the SgBP for the Plurality rule given the number of voters. He found that the probability grows from $1.90 \%$ with nine voters to $2.78 \%$ when the electorate tends to infinity. Gehrlein and Lepelley (2010b) have provided a general formula but not too easy to handle. We enrich the results of Lepelley (1993) by showing that: with three candidates and preferences consistent with the NBR, there is no scoring rule such that the Condorcet loser scores better than the never bottom-ranked candidate; moreover, it is only under the Antiplurality rule

\footnotetext{
${ }^{5}$ Scoring rules are voting systems that give points to candidates according to the position they have in voters' rankings. The winner is the candidate with the highest total of points received.

${ }^{6}$ With $m$ candidates, the Borda rule is a scoring rule that gives $m-1$ points to a candidate when she is ranked first, $m-2$ when she is ranked second, so and so, and 0 when she is ranked last.

${ }^{7}$ See Saari (1995), Tataru and Merlin (1997), Lepelley et al. (2000a), Gehrlein (2002), Gehrlein and Lepelley (2010a), Diss and Gehrlein (2012) for more on this.

${ }^{8}$ The Antiplurality rule or Negative Plurality elects the candidate with the fewest number of last positions.
} 
that the Condorcet loser always scores the worst. We also provide a more tractable formula of the likelihood of the SgBP for any number of voters.

Under the NTR, Lepelley et al. (2000b), Gehrlein and Lepelley (2010b) analyzed the SgBP by focusing on the following scoring rules : the Borda rule, the Plurality rule and the Antiplurality rule. It came out that the $S g B P$ never occurs with the Plurality rule and the Borda rule. In this paper, we supplement this result. We use an analytical approach to show that with three candidates, the Strong Borda Paradox never occurs with the NTR for all the scoring rules located between the Plurality rule and the Borda rule. Gehrlein and Lepelley (2010b) provided a formula for the likelihood of the SgBP under Antiplurality rule for a various number of voters under the NTR. This formula is a bit hard to deal with. Here, we provide a more attractive formula. We also show that i) if the Condorcet loser is not the never topranked candidate, the Antiplurality rule is the only scoring rule that always ranks this candidate last; ii) if the Condorcet loser is the never top-ranked candidate, she is always ranked last for all the scoring rules located between the Plurality rule and the Borda rule.

Only a little attention has been paid to the NMR when analyzing the Strong Borda Paradox $^{9}$. In this paper, we use an analytical approach to find out all the scoring rules that are vulnerable to the Strong Borda Paradox under the NMR. We find that in three-candidate elections, if there is a Condorcet loser, except for the Borda rule, all the scoring rules are vulnerable to the Strong Borda Paradox. We Compute, given the number of voters, the likelihood of the Strong Borda Paradox for the Plurality rule and the Antiplurality rule under this restriction. We also show that if the Condorcet loser is not the never middle-ranked candidate, the Borda rule is the only scoring rule that always ranks this candidate last; if the Condorcet loser is the never middle-ranked candidate, the Antiplurality rule is the only scoring rule that always ranks her last.

The rest of the paper is organized as follows: Section 2 is devoted to the basic notation and the definitions. Section 3 presents our results derived from the analytical approach. Even if the NBR, NTR and the NMR guarantee the existence of a Condorcet winner, it is not clear a priori that such restrictions are likely to reduce the likelihood of voting paradoxes. The probability computations of Section 4 will tell us more on this. Section 5 concludes.

\footnotetext{
${ }^{9}$ Concerning the NMR, Gehrlein (2006) focused on the probability of accordance between the majority rule and a scoring rule given that there is a Condorcet winner.
} 


\section{Basic Framework}

\section{$2.1 \quad$ Preferences}

Let $N$ be the set of $n$ voters, $n \geq 2$ and $A$ the set of $m \geq 3$ candidates. A binary relation $R$ on $A$ is a subset of the cartesian product $A \times A$. For $a, b \in A$, if $(a, b) \in R$, we note $a R b^{10}$ to say that " $a$ is at least as good as $b$ ". $\neg a R b$ is the negation of $a R b$. If we have $a R b$ and $\neg b R a$, then we say that " $a$ is better than or strictly preferred to $b$ ". In that case, we note $a P b$ with $P$ the asymmetric component of $R$. $I$ the symmetric component of $R$, is defined by $a I b$ translating an indifference between $a$ and $b$ i.e $\neg a R b$ and $\neg b R a$. The preference profile $\pi=\left(\pi_{1}, \pi_{2}, \ldots, \pi_{i}, \ldots, \pi_{n}\right)$ gives the linear orders ${ }^{11}$ of the $n$ voters on $A$ where $\pi_{i}$ is the strict ranking of voter $i$. The set of all preference profiles on $A$ is $P(A)^{n}$. In our framework, voters are supposed to have strict rankings only.

With $n_{t}$ and $p_{t}=\frac{n_{t}}{n}$ respectively the number and the proportion of voters with type $t$, a voting situation is defined by $\tilde{n}=\left(n_{1}, \ldots, n_{t}, \ldots, n_{m !}\right)$ such that $\sum_{t=1}^{m !} n_{t}=n$. With vector the $p=\left(p_{1}, \ldots, p_{t}, \ldots, p_{m !}\right)\left(p \in \mathbb{R}^{m !}\right)$, the domain of the voting rules can be represented by the points of the unit simplex $S(m !)$ defined by:

$$
S(m !)=\left\{p \in \mathbb{R}^{m !}: \sum_{t=1}^{m !} p_{t}=1, p_{t} \geq 0\right\}
$$

Table 1 lists the six strict rankings or possible types of strict preferences on $A=$ $\{a, b, c\}$.

Table 1: Possible preferences types on $A=\{a, b, c\}$

\begin{tabular}{||c|c||c|c||}
\hline type $t$ & preferences & type $t$ & preferences \\
\hline 1 & $a P b P c$ & 4 & $c P b P a$ \\
2 & $a P c P b$ & 5 & $b P c P a$ \\
3 & $c P a P b$ & 6 & $b P a P c$ \\
\hline
\end{tabular}

\footnotetext{
${ }^{10}$ When the binary relation $R$ is used for a particular voter, we write $a R_{i} b$ to say that voter $i$ finds " $a$ at least as good as $b$ ". If there is no particular mark under $R$, it means that $R$ is a collective preference ranking. This will remain valid for relations $P$ and $I$.

${ }^{11}$ A linear order is a binary relation that is transitive, complete and antisymmetric. The binary relation $R$ on $A$ is transitive if for $a, b, c \in A$, if $a R b$ and $b R c$ then aRc. $R$ is antisymmetric if for all for $a \neq b, a R b \Rightarrow \neg b R a$; if we have $a R b$ and $b R a$, then $a=b . R$ is complete if and only if for all $a, b \in A$, we have $a R b$ or $b R a$.
} 


\subsection{Scoring rules}

Scoring rules are voting systems that give points to candidates according to the position they have in voters' ranking. In general, with $m \geq 3$ and strict rankings, $w=$ $\left(w_{1}, w_{2}, \ldots, w_{k}, \ldots, w_{m}\right)$ is a vector in $\mathbb{R}^{m}$, such that $w_{k}$ points ( $w_{k}$ is a real number) are given to a candidate when she appears ranked $k$-th in a voter's preference; with $w_{j} \geq w_{k}(j \leq k)$ and $w_{1}>w_{m}$. The score of a candidate is the total number of points awarded by the voters to this candidate. The winner is the one with the highest score.

In the unit simplex $S(m$ !), a normalized scoring vector can be used. With three candidates, a normalized scoring vector for the family of simple positional rules in $S(3 !)$ is $w_{\lambda}=(1, \lambda, 0)$ with $0 \leq \lambda \leq 1$. If $\lambda=0$, the voting rule is the simple Plurality

rule. For $\lambda=1$, we have the Antiplurality rule and for $\lambda=\frac{1}{2}$, we have the Borda rule. Without ambiguity, we denote by $w_{\lambda}(a)$ the score of candidate $a$ given $\lambda$. Table 2 gives the scores of candidates on $A=\{a, b, c\}$ using the labels of Table 1.

Table 2: Candidates' scores on $A=\{a, b, c\}$

$$
\begin{aligned}
& w_{\lambda}(a)=n_{1}+n_{2}+\lambda\left(n_{3}+n_{6}\right) \\
& w_{\lambda}(b)=n_{5}+n_{6}+\lambda\left(n_{1}+n_{4}\right) \\
& w_{\lambda}(c)=n_{3}+n_{4}+\lambda\left(n_{2}+n_{5}\right)
\end{aligned}
$$

\subsection{Preference restrictions}

When dealing with each of our restrictions, it is no longer possible to treat the candidates symmetrically as in the universal domain. By definition, the never bottomranked candidate seems to have an advantage while the never top-ranked candidate is disadvantaged in a certain way. In the sequel, for each of our restrictions, we will just assume a candidate on whom the supposed restriction is fulfilled. The reasoning on the other candidates follows a similar scheme.

With the well-known NBR, there is a candidate that is never bottom ranked by any voter. Let us assume on $A=\{a, b, c\}$ that this is candidate $c$; this leads to the deletion of types 1 and 6 in Table 1 . We end up with the voting situation described in Table 3.

As the NMR requires that there is a candidate (assume $c$ ) that never appears middle ranked, this leads to the deletion of types 2 and 5 and we end up with Table 4 . 
Table 3: Possible preference types on $A=\{a, b, c\}$ with respect to the NBR

\begin{tabular}{||c|c||}
\hline type $t$ & preferences \\
\hline 2 & $a P c P b$ \\
\hline 3 & $c P a P b$ \\
\hline 4 & $c P b P a$ \\
\hline 5 & $b P c P a$ \\
\hline
\end{tabular}

$$
\begin{gathered}
\underline{\text { Scores }} \\
w_{\lambda}(a)=n_{2}+\lambda n_{3} \\
w_{\lambda}(b)=n_{5}+\lambda n_{4} \\
w_{\lambda}(c)=n_{3}+n_{4}+\lambda\left(n_{2}+n_{5}\right)
\end{gathered}
$$

Table 4: Possible preference types on $A=\{a, b, c\}$ with respect to the NMR

\begin{tabular}{||c|c||}
\hline type $t$ & preferences \\
\hline 1 & $a P b P c$ \\
\hline 3 & $c P a P b$ \\
\hline 4 & $c P b P a$ \\
\hline 6 & $b P a P c$ \\
\hline
\end{tabular}

$$
\begin{gathered}
\text { Scores } \\
w_{\lambda}(a)=n_{1}+\lambda\left(n_{3}+n_{6}\right) \\
w_{\lambda}(b)=n_{6}+\lambda\left(n_{1}+n_{4}\right) \\
w_{\lambda}(c)=n_{3}+n_{4}
\end{gathered}
$$

In such a case, candidate $c$ appears as a polarizing candidate because she cuts the electorate into two contrasting positions (Gehrlein, 2006).

The NTR requires that there is a candidate (assume $c$ ) that no voter ranks at the top of his ranking. This implies the deletion of types 3 and 4 and we end up with Table 5 .

Table 5: Possible preference types on $A=\{a, b, c\}$ with respect to the NTR

\begin{tabular}{||c|c||}
\hline type $t$ & preferences \\
\hline 1 & $a P b P c$ \\
\hline 2 & $a P c P b$ \\
\hline 5 & $b P c P a$ \\
\hline 6 & $b P a P c$ \\
\hline
\end{tabular}

$$
\begin{gathered}
\stackrel{\text { Scores }}{n_{1}+n_{2}+\lambda n_{6}} \\
w_{\lambda}(a)=n_{5}+n_{6}+\lambda n_{1} \\
w_{\lambda}(b)=n_{5}(c)=\lambda\left(n_{2}+n_{5}\right) \\
w_{\lambda}(c)
\end{gathered}
$$




\subsection{The pairwise comparisons}

If the number of voters who rank candidate $a$ before candidate $b$ is greater than that of those who rank candidate $b$ before candidate $a$, then candidate $a$ is said to be majority preferred to candidate $b$. If candidate $a$ is majority preferred to any other candidate in $A, a$ is called the Condorcet winner. If each candidate in $A \backslash\{a\}$ is majority preferred to $a$, then candidate $a$ is the Condorcet loser.

Suppose that $a$ is the Condorcet loser for each of the restrictions. Formally, we get Equation (2.1) with the $N B R$, Equation (2.2) with the $N M R$ and Equation (2.3) for the NTR.

$$
\begin{aligned}
& \left\{\begin{array}{l}
n_{4}+n_{5}>n_{2}+n_{3} \\
n_{3}+n_{4}+n_{5}>n_{2}
\end{array}\right. \\
& \left\{\begin{array}{l}
n_{4}+n_{6}>n_{1}+n_{3} \\
n_{3}+n_{4}>n_{1}+n_{6}
\end{array}\right. \\
& \left\{\begin{array}{l}
n_{5}+n_{6}>n_{1}+n_{2} \\
n_{5}>n_{1}+n_{2}+n_{6}
\end{array}\right.
\end{aligned}
$$

One can notice in Equation 2.1 that the first inequality implies the second one; in Equation 2.3, the second inequality implies the first one second. Due to the fact that candidates cannot be treated symmetrically, if $b$ or $c$ is assumed to be the Condorcet loser, one will get inequalities totally different from those we get by assuming that $a$ is the Condorcet loser ${ }^{12}$.

Recall that the aim of this paper is to find out all the scoring rules that can elect the Condorcet loser for each kind of preference restrictions.

\section{Preference restrictions, scoring rules and the elec- tion of the Condorcet loser}

This section will let us know for each of our preference restrictions, which scoring rules are vulnerable to the strong Borda Paradox in three-candidate elections. For the NBR, we first recall the already known results on the issue and then, we analyze the other scenarios.

Again, we will denote by $c$ the candidate on whom the restriction is satisfied and by $a$ the candidate on whom it is not. For our analysis, we will distinguish the voting

\footnotetext{
${ }^{12}$ The reader can easily get these inequalities.
} 
situations where the Condorcet loser is candidate $a$ from those she is candidate $c$. Such a distinction cannot be made with three candidates under the NBR restriction since the never bottom-ranked candidate cannot be a Condorcet loser.

\subsection{The NBR and the Strong Borda Paradox}

In three-candidate elections, Lepelley (1993) showed that the Antiplurality rule never elects the Condorcet loser under the NBR restriction. Lepelley et al. (2000b) (see also Gehrlein and Lepelley (2010a)) computed the likelihood of the SgBP for all the scoring rules under this restriction. From their computations, one can derive what follows.

Theorem 1. (Lepelley et al., 2000b)In three-candidate elections, the Condorcet loser can be elected for all $\lambda \in\left[0 \frac{1}{2}\right.$ [ under the NBR restriction. This is never the case for all $\lambda \in\left[\frac{1}{2} 1\right]$.

According to Theorem 1, with three candidates, the SgBP never occurs with all the scoring rules located between the Antiplurality rule and the Borda rule. Lemma 1 tells us that in three-candidate elections, it is only under the Antiplurality rule that this candidate always performs the worst.

Lemma 1. Consider a voting situation with three candidates where preferences are consistent with the NBR restriction. For $\lambda \in\left[\begin{array}{ll}0 & 1\end{array}\right]$, the Condorcet loser never scores better than the never bottom-ranked candidate. Except for the Antiplurality rule, there is no other scoring rule under which the Condorcet loser always gets the lowest score.

Proof. As we know that with three candidates, the never bottom-ranked candidate cannot be a Condorcet loser under the NBR restriction, let us assume that candidate $a$ is the Condorcet loser. By Theorem 1, this candidate can be ranked first for $\lambda \in\left[0 \frac{1}{2}\right.$ [ but never for $\lambda \in\left[\frac{1}{2} 1\right]$. So, for the proof of the lemma, we only need to consider $\lambda \in\left[\frac{1}{2} 1\right]$. Without loss of generality, let us consider $\lambda=1$. It follows that

$$
w_{1}(a)-w_{1}(b)=n_{2}+n_{3}-n_{4}-n_{5} \quad \text { and } \quad w_{1}(a)-w_{1}(c)=-n_{4}-n_{5}
$$

It comes that $w_{1}(a)-w_{1}(b)<0$ and $w_{1}(a)-w_{1}(c)<0$ : so, candidate $a$ is always ranked last. Let us now consider $\lambda \in\left[\frac{1}{2} 1\left[\right.\right.$. Suppose that $w_{\lambda}(a)>w_{\lambda}(c)$. So we have,

(i) $\quad w_{\lambda}(a)-w_{\lambda}(c)=(\lambda-1) n_{2}+(1-\lambda) n_{3}+n_{4}+\lambda n_{5}<0$

(ii) $\quad n_{2}-n_{4}-n_{5}<0 \quad$ by Equation (2.1) 
We get by $(i)+(i i) \Leftrightarrow \lambda n_{2}+(1-\lambda) n_{3}+\lambda n_{5}<0$ : contradiction. So, for $\lambda \in\left[\frac{1}{2} 1\right]$, we never have $w_{\lambda}(a)>w_{\lambda}(c)$. It follows that our proof will then consist in showing that there is always a profile and a $\lambda \in\left[\frac{1}{2} 1\right.$ [ such that candidate $a$ can do better than candidate $b$. For $\lambda \in\left[\frac{1}{2} 1\right.$ [, let us take $n_{2}=3, n_{3}=0, n_{4}=2$ and $n_{5}=2$. The reader can easily check that candidate $a$ is the Condorcet loser. Also, we have $w_{\lambda}(a)-w_{\lambda}(b)=1-\lambda>0$. So, the Condorcet loser $a$ performs better than candidate $b$ for $\lambda \in\left[\frac{1}{2} 1\right.$. Thus, with the NBR restriction, it is only under the Antiplurality rule that the Condorcet loser performs the worst.

\subsection{The NTR and the Strong Borda Paradox}

Under the NTR restriction, Theorem 2 tell us that in three-candidate elections, the Condorcet loser is can be elected for all the scoring rules located between the Borda rule and the Antiplurality rule.

Theorem 2. With three candidates, a Condorcet loser can be elected for $\left.\lambda \in] \frac{1}{2} 1\right]$ under the NTR restriction; this is never the case for $\lambda \in\left[\begin{array}{ll}0 & \frac{1}{2}\end{array}\right]$.

The proof of Theorem 2 comes as a conclusion from Lemmata 2 and 3.

Lemma 2. Let $A=\{a, b, c\}$ and the NTR restriction made with respect to candidate c. If one candidate in $A \backslash\{c\}$ is the Condorcet loser, she is never elected for no scoring rule. Except for the Antiplurality rule, there is no other scoring rule that always ranks this candidate last.

Proof. Let us consider a voting situation with $A=\{a, b, c\}$ and the NTR with respect to candidate $c$. Without loss of generality, suppose that $a$ is the Condorcet loser. So, Equation (2.3) holds. We have

$$
w_{\lambda}(a)-w_{\lambda}(b)=(1-\lambda) n_{1}+n_{2}-n_{5}+(\lambda-1) n_{6}
$$

By Equation (2.3), $n_{5}>n_{1}+n_{2} \Rightarrow n_{5}>(1-\lambda) n_{1}+n_{2}$. Since $(\lambda-1) n_{6} \leq 0$ for all $\lambda \in\left[\begin{array}{ll}0 & 1\end{array}\right]$, it comes that $w_{\lambda}(a)<w_{\lambda}(b)$ for all $\lambda \in\left[\begin{array}{ll}0 & 1\end{array}\right]$. So, candidate $b$ always scores better than candidate $a$ : with the NTR, if one candidate in $A \backslash\{c\}$ is the Condorcet loser, she is never elected for no scoring rule. Now, let us show that the Antiplurality rule is the only rule that always ranks this candidate last. For this, it needs to show that except for the Antiplurality rule, it is always possible to exhibit a profile under which the Condorcet loser candidate $a$ can do better than the never top-ranked candidate $c$. First, for $\lambda=1$, we have $w_{1}(a)-w_{1}(c)=n_{1}+n_{6}-n_{5}<0$; thus $w_{1}(a)<w_{1}(c)$ : candidate $a$ is ranked last for the Antiplurality rule. For 
$\lambda \in\left[01\right.$ [ assume a profile where $n_{1}=z-1, n_{2}=n_{6}=0$ and $n_{5}=z$ with $z>1$. We have, $w_{\lambda}(a)-w_{\lambda}(c)=(1-\lambda) z-1>0$. It follows that given $\lambda$, it is always possible to find $z>\left\lceil\frac{1}{1-\lambda}\right\rceil$ such that $w_{\lambda}(a)>w_{\lambda}(c)$. So, it is always possible to get a profile such that candidate $a$ scores better than candidate $c$. Thus, with the NTR, except for $\lambda=1$, there is no other $\lambda$ such that a Condorcet loser in $A \backslash\{c\}$ always scores the worst.

Lemma 3. Let $A=\{a, b, c\}$ and the NTR restriction made with respect to candidate c. If candidate $c$ is the Condorcet loser, she can be elected for $\left.\lambda \in] \frac{1}{2} 1\right]$. For $\lambda \in\left[0 \frac{1}{2}\right]$, she is never elected and is always ranked last.

Proof. Consider a three-candidate voting situation under the NTR. Suppose that candidate $c$ is the Condorcet loser; this means that $n_{1}+n_{2}+n_{6}>n_{5}$ (i) and $n_{1}+n_{5}+n_{6}>n_{2}$ (ii). We get

$$
w_{\lambda}(b)-w_{\lambda}(c)=n_{5}+n_{6}+\lambda n_{1}-\lambda n_{2}-\lambda n_{5}=\lambda n_{1}-\lambda n_{2}+(1-\lambda) n_{5}+n_{6}
$$

For $\lambda \in\left[0 \frac{1}{2}\right]$, it is obvious that $\lambda n_{1}-\lambda n_{2}+(1-\lambda) n_{5}+n_{6}>\lambda\left(n_{1}-n_{2}+n_{5}+n_{6}\right)>0$ (by (ii)); then, $w_{\lambda}(b)>w_{\lambda}(c)$. Moreover,

$$
w_{\lambda}(a)-w_{\lambda}(c)=n_{1}+n_{2}+\lambda n_{6}-\lambda n_{2}-\lambda n_{5}=n_{1}+(1-\lambda) n_{2}-\lambda n_{5}+\lambda n_{6}
$$

For $\lambda \in\left[0 \frac{1}{2}\right]$, it is obvious that $n_{1}+(1-\lambda) n_{2}-\lambda n_{5}+\lambda n_{6}>\lambda\left(n_{1}+n_{2}-n_{5}+n_{6}\right)>0$ (by (i)); then, $w_{\lambda}(a)>w_{\lambda}(c)$. Thus, candidate $c$ is never elected and is always ranked last for $\lambda \in\left[\begin{array}{ll}0 & \frac{1}{2}\end{array}\right]$. To show that candidate $c$ can be elected for $\left.\left.\lambda \in\right] \frac{1}{2} 1\right]$, we just consider a profile with $n_{1}=n_{6}=1$ and $n_{2}=n_{5}=z$ such that $z=\left\lceil\frac{1+\lambda}{2 \lambda-1}\right\rceil$; such a $z$ always exists given $\left.\lambda \in] \frac{1}{2} 1\right]$.

Thus, under the NTR, the Strong Borda Paradox can occur for all the scoring rules such that $\left.\lambda \in] \frac{1}{2} 1\right]$.

\subsection{The NMR and the Strong Borda Paradox}

According to Theorem 3 that follows, Borda rule is the only scoring rule that is free from the Strong Borda Paradox in three-candidate elections when preferences satisfied the NMR.

Theorem 3. With three candidates and the NMR restriction, except for the Borda rule there is no other scoring rule that prevents the election of the Condorcet loser.

The proof of Theorem 3 comes from a juxtaposition of Lemmata 4 and 5 that follow. 
Lemma 4. Let $A=\{a, b, c\}$ and the NMR restriction is made with respect to candidate c. If a candidate in $A \backslash\{c\}$ is the Condorcet loser, she can be elected for $\left.\lambda \in] \frac{1}{2} 1\right]$. Except for the Borda rule, no other scoring rule ensures that she is always ranked last.

Proof. Consider a three-candidate elections under NMR and suppose that candidate $a$ is the Condorcet loser. Assume $\lambda=\frac{1}{2}-\epsilon$ such that $0<\epsilon \leq \frac{1}{2}$. We have

$$
\begin{aligned}
w_{\lambda}(a)-w_{\lambda}(c) & =n_{1}+\left(\frac{1}{2}-\epsilon\right)\left(n_{3}+n_{6}\right)-\left(n_{3}+n_{4}\right) \\
& =\frac{1}{2} \underbrace{\left(n_{1}-n_{4}\right)}_{<0 \text { by Equation }(2.1)}+\frac{1}{2} \underbrace{\left(n_{1}-n_{3}-n_{4}+n_{6}\right)}_{<0 \text { by Equation }(2.2)} \underbrace{-\epsilon\left(n_{3}+n_{6}\right)}_{<0}
\end{aligned}
$$

Then, for $\lambda \in\left[\begin{array}{ll}0 & \frac{1}{2}\end{array}\right]$, we always have $w_{\lambda}(a)<w_{\lambda}(c)$ : the Condorcet loser, candidate $a$, is never elected since she always scores less than the never middleranked candidate $c$. Let us show that over this range, the Condorcet loser is always ranked last only with the Borda rule $\left(\lambda=\frac{1}{2}\right)$. For $\lambda=\frac{1}{2}$,

$$
\begin{aligned}
& w_{\lambda}(a)-w_{\lambda}(b)=\frac{1}{2}\left(n_{1}+n_{3}-n_{4}-n_{6}\right)<0 \text { by Equation }(2.2) \\
& w_{\lambda}(a)-w_{\lambda}(c)=\frac{1}{2}\left(n_{1}-n_{4}\right)+\frac{1}{2}\left(n_{1}-n_{3}-n_{4}+n_{6}\right)<0 \text { by Equation }(2.2)
\end{aligned}
$$

So, for $\lambda=\frac{1}{2}$, we always have $w_{\lambda}(a)<w_{\lambda}(b)$ and $w_{\lambda}(a)<w_{\lambda}(c)$ : the Condorcet loser is always ranked last. As we have just shown that candidate $a$ always scores less than candidate $c$ for all $\lambda \in\left[\begin{array}{ll}0 & \frac{1}{2}\end{array}\right]$, let us take $\lambda \in\left[0 \frac{1}{2}[\right.$ and show that we can find a profile such that $w_{\lambda}(a)>w_{\lambda}(b)$. For this, consider the following profile : $n_{1}=n_{4}=3, n_{3}=2$ and $n_{6}=1$; it is clear that candidate $a$ is the Condorcet loser in this profile. The scores are $w_{\lambda}(a)=3(1+\lambda), w_{\lambda}(b)=1+6 \lambda$ and $w_{\lambda}(c)=5$. We can easily check that $w_{\lambda}(a)-w_{\lambda}(b)=2-3 \lambda>0$ for all $\lambda \in\left[0 \quad \frac{1}{2}[\right.$. To show that candidate $a$ can be elected for $\lambda \in] \frac{1}{2} 1$ ], just assume a profile such that $n_{1}=1$, $n_{3}=n_{6}=z$ and $n_{4}=2$ with $z=\left\lceil\frac{1}{2 \lambda-1}\right\rceil$.

Lemma 5. Let assume on $A=\{a, b, c\}$, the NMR restriction with respect to candidate $c$. If candidate $c$ is the Condorcet loser, she can be elected for $\lambda \in\left[0 \frac{1}{2}[\right.$. Except for the Antiplurality rule, no other scoring rule ensures that she always scores the worst.

Proof. Consider a three-candidate election under NMR and suppose that $c$ is the Condorcet loser. This means that $n_{1}+n_{6}>n_{3}+n_{4}$. As we know that the Borda 
rule never elects the Condorcet loser, we have $w_{\frac{1}{2}}(a)-w_{\frac{1}{2}}(c)>0$ or $w_{\frac{1}{2}}(b)-w_{\frac{1}{2}}(c)>0$ for a given profile. Let us assume $\lambda=\frac{1}{2}+\epsilon$ with $0<\epsilon \leq \frac{1}{2}$. In such a case, we have

$$
\left\{\begin{array}{l}
w_{\frac{1}{2}+\epsilon}(a)=w_{\frac{1}{2}}(a)+\epsilon\left(n_{3}+n_{6}\right)>w_{\frac{1}{2}}(a) \\
w_{\frac{1}{2}+\epsilon}(b)=w_{\frac{1}{2}}(b)+\epsilon\left(n_{1}+n_{4}\right)>w_{\frac{1}{2}}(b) \\
w_{\frac{1}{2}+\epsilon}(c)=w_{\frac{1}{2}}(c)
\end{array}\right.
$$

Since candidate $c$ is not elected for $\lambda=\frac{1}{2}$, according to Equation (3.1), she is still not elected for $\lambda=\frac{1}{2}+\epsilon$. Thus, the Condorcet loser is never elected for $\lambda \in\left[\begin{array}{ll}\frac{1}{2} & 1\end{array}\right]$.

We are going now to show over the range $\lambda \in\left[\begin{array}{ll}\frac{1}{2} & 1\end{array}\right]$ that only the Antiplurality ensures that the Condorcet loser is always ranked last. For $\lambda=1$, we have $w_{1}(a)=$ $n_{1}+n_{3}+n_{6}, w_{1}(a)=n_{1}+n_{4}+n_{6}$ and $w_{1}(c)=n_{3}+n_{4}$. Since $n_{1}+n_{6}>n_{3}+n_{4}$, it follows that $w_{1}(a)>w_{1}(c)$ and $w_{1}(b)>w_{1}(c)$ : the Condorcet loser $c$ scores the worst under the Antiplurality. Now, suppose $\lambda \neq 1$; for $\lambda \in\left[\frac{1}{2} 1\right.$ [, assume a profile such that $n_{1}=z+1, n_{3}=z, n_{4}=1$ and $n_{6}=1$ with $z=\left\lceil\frac{2}{1-\lambda}\right\rceil$. It is easy to check that with this profile, candidate $c$ is the Condorcet loser and that $w_{\lambda}(a)>w_{\lambda}(c)$ and $w_{\lambda}(b)<w_{\lambda}(c)$. Thus, for $\lambda \in\left[\frac{1}{2} 1\right.$, it is always possible to find a profile such that the Condorcet loser is not ranked last. It is always possible, for $\lambda \in\left[\begin{array}{l}0 \\ \frac{1}{2}\end{array}\right.$, to find a profile under which the Condorcet loser can be the winner.

Table 6: Preference restrictions and Scoring rules that can elect the Condorcet loser in three-candidate elections

\begin{tabular}{|c|c|c|}
\hline \multicolumn{3}{|c|}{ Preference restrictions } \\
\hline $\mathrm{NBR}^{*}$ & NMR & NTR \\
\hline$\lambda \in\left[\begin{array}{ll}0 & \frac{1}{2}\end{array}\right.$ & $\lambda \in] 0 \quad \frac{1}{2}[\cup] \frac{1}{2}$ & $\left.\lambda \in] \frac{1}{2} \quad 1\right]$ \\
\hline
\end{tabular}

* From Lepelley et al. (2000b)

Tables 6 and 7 summarize all the above results. Table 7 lists for each of our restrictions, the scoring rules under which the Condorcet loser always scores less than the two other candidates. Table 6 gives all the scoring rules that are vulnerable to the Strong Borda Paradox for each of our restrictions. It is well-known that the Borda rule is immune to the Strong Borda Paradox; for the other values of $\lambda$ one can find a situation under which the paradox occurs. For example, we have learnt 
Table 7: Preference restrictions and Scoring rules that always rank the Condorcet loser last in three-candidate elections

\begin{tabular}{||c||c||c||c||}
\hline \hline \multicolumn{1}{||c||}{} & \multicolumn{3}{c||}{ Prefence restrictions } \\
\hline Condorcet loser & NBR & NMR & NTR \\
\hline \hline candidate $a$ & $\lambda=1$ & $\lambda=\frac{1}{2}$ & $\lambda=1$ \\
\hline \hline candidate $c$ & - & $\lambda=\frac{1}{2}$ & $\lambda \in\left[\begin{array}{ll}0 & \frac{1}{2}\end{array}\right]$ \\
\hline \hline
\end{tabular}

that the Plurality rule is vulnerable to the Strong Borda Paradox under the NBR and the Antiplurality rule is vulnerable under the NMR and the NTR.

Even if the NBR, the NTR and the NMR guarantee the existence of a Condorcet winner, it is not clear if such restrictions are likely to reduce the likelihood of voting paradoxes. Probability computations will allow us to know more on this.

\section{The preference restrictions and the probability of electing the Condorcet loser}

Gehrlein and Lepelley (2010b) have provided a more general formula but not too easy to handle. This is also the case for the formula of the likelihood of the SgBP under the NTR they suggested. Here, we will focus on the Plurality rule and the Antiplurality rule and provide more tractable formulas of the likelihood of the SgBP for all number of voters. We perform our computations under the Impartial Anonymous Culture (IAC). The IAC is one of the most used probability assumptions in the social choice literature when computing the likelihood of voting events. This assumption was first introduced by Gehrlein and Fishburn (1976). Under IAC, each voting situation is equally likely. The likelihood of a given event $X$ is calculated in respect with the following ratio:

$$
P(X)=\frac{\text { Number of voting situations in which event } X \text { occurs }}{\text { Total number of possible voting situations }}
$$

There are many algorithms or techniques in the literature that enable the calculation of the number of voting situations in which an event $X$ occur. Without been exhaustive, we can list among others, Barvinok (1994), Bruynooghe et al. (2005), Clauss and Loechner (1998), Barvinok and Pommersheim (1999), Gehrlein and Fishburn (1976), Huang and Chua (2000), Lepelley et al. (2008), Wilson and Pritchard 
(2007). In this paper, we use the parameterized Barvinok's algorithm developed by Verdoolaege et al. (2004). This algorithm proceeds by the enumeration of the Ehrhart's polynomials (Ehrhart, 1962, 1967). For more on the Ehrhart's theory, the reader can refer to Ehrhart $(1962,1967)$, Barvinok (1994), Barvinok and Pommersheim (1999), Bruynooghe et al. (2005), Clauss and Loechner (1998), Verdoolaege et al. (2004).

So, given $n$ the size of the electorate, we compute $P_{\mathrm{NBR}}^{\mathrm{PR}}(3, n, \mathrm{IAC}), P_{\mathrm{NTR}}^{\mathrm{APR}}(3, n, \mathrm{IAC})$, $P_{\mathrm{NMR}}^{\mathrm{PR}}(3, n, \mathrm{IAC})$ and $P_{\mathrm{NMR}}^{\mathrm{APR}}(3, n, \mathrm{IAC})$ the likelihood of the Strong Borda Paradox under each of the restrictions for the Plurality rule $(\mathrm{PR})$ and the Antiplurality rule (APR). We report all the formulas of these probabilities in the Appendix (see Formulas 1 to 4 ).

In Table 8, we report some values of the likelihood of the Strong Borda Paradox under each of our restrictions for the Plurality rule and the Antiplurality rule. We also report the likelihood of the Strong Borda Paradox without restrictions under the Plurality rule $\left(P^{\mathrm{PR}}(3, n, \mathrm{IAC})\right)$ and the Antiplurality rule $\left(P^{\mathrm{APR}}(3, n, \mathrm{IAC})\right)$.

Table 8: Preference restrictions and probability of the Strong Borda Paradox

\begin{tabular}{|c|c|c|c|c|c|c|}
\hline$n$ & $P_{\mathrm{NBR}}^{\mathrm{PR}}(3, n, \mathrm{IAC})$ & $P_{\mathrm{NTR}}^{\mathrm{APR}}(3, n, \mathrm{IAC})$ & $P_{\mathrm{NMR}}^{\mathrm{PR}}(3, n, \mathrm{IAC})$ & $P_{\mathrm{NMR}}^{\mathrm{APR}}(3, n, \mathrm{IAC})$ & $P^{\mathrm{PR}}(3, n, \mathrm{IAC})^{*}$ & $P^{\mathrm{APR}}(3, n, \mathrm{IAC})^{*}$ \\
\hline 5 & & 0.0400 & & 0.0400 & 0.0091 & 0.0199 \\
\hline 6 & & 0 & & 0 & 0.0113 & 0.0200 \\
\hline 7 & 0.0093 & 0.0179 & 0.0357 & 0.0179 & 0.0128 & 0.0203 \\
\hline 8 & 0.0038 & 0.0192 & 0 & 0.0128 & 0.0140 & 0.0206 \\
\hline 9 & 0.0198 & 0.0381 & 0.0476 & 0.0381 & 0.0149 & 0.0209 \\
\hline 10 & 0.0038 & 0.0109 & 0.0182 & 0.0073 & 0.0158 & 0.0213 \\
\hline 11 & 0.0206 & 0.0341 & 0.0511 & 0.0341 & 0.0165 & 0.0217 \\
\hline 12 & 0.0094 & 0.0249 & 0.0271 & 0.0181 & 0.0171 & 0.0220 \\
\hline 15 & 0.0227 & 0.0375 & 0.0675 & 0.0375 & 0.0186 & 0.0229 \\
\hline 20 & 0.0148 & 0.0326 & 0.0497 & 0.0263 & 0.0203 & 0.0241 \\
\hline 33 & 0.0254 & 0.0376 & 0.0886 & 0.0326 & 0.0226 & 0.0258 \\
\hline 50 & 0.0218 & 0.0412 & 0.0826 & 0.0375 & 0.0242 & 0.0269 \\
\hline 81 & 0.0267 & 0.0465 & 0.1013 & 0.0465 & 0.0254 & 0.0278 \\
\hline 100 & 0.0246 & 0.0449 & 0.0962 & 0.0427 & 0.0258 & 0.0281 \\
\hline 117 & 0.0271 & 0.0471 & 0.1042 & 0.0471 & 0.0261 & 0.0283 \\
\hline 305 & 0.0275 & 0.0480 & 0.1084 & 0.0480 & 0.0271 & 0.0290 \\
\hline$\infty$ & 0.0278 & 0.0486 & 0.1111 & 0.0486 & 0.0278 & 0.0295 \\
\hline
\end{tabular}

It comes from Table 8 that the vulnerability of the Antiplurality to the Strong Borda Paradox tends to be the same for some values of $n$ under the NTR and the NMR. For example, this is the case for $n=5, n=6, n=9$ and when the electorate is infinite. We also notice that for the Plurality rule, the Strong Borda Paradox tends 
to be more likely under the NMR; for the Antiplurality rule, it is under the NTR. For each restriction, the probabilities tend to increase with the number of voters as it is the case when no restriction is assumed.

The interesting question is the following: when a restriction does not prevent the paradox to happen, can we at least expect a reduction in the probability of this paradox? Concerning the Plurality rule and the Antiplurality rule, the answer follows.

- With the Plurality rule : we have to compare $P_{\mathrm{NBR}}^{\mathrm{PR}}(3, n, \mathrm{IAC})$ and $P_{\mathrm{NMR}}^{\mathrm{PR}}(3, n, \mathrm{IAC})$ to $P^{\mathrm{PR}}(3, n, \mathrm{IAC})$. We clearly notice that the NMR does not reduce the likelihood of the Strong Borda Paradox; with more than 8 voters, the probability of the paradox under the NMR given $n$ is almost 2 to 5 times greater than that with no restriction. Concerning the impact of the NBR, nothing a priori can be said as the dominance between $P_{\mathrm{NBR}}^{\mathrm{PR}}(3, n, \mathrm{IAC})$ and $P^{\mathrm{PR}}(3, n, \mathrm{IAC})$ alternates with a value of $n$ to another. However, we note that the probability is the same when the electorate is infinite.

- With the Antiplurality rule. By comparing $P_{\mathrm{NTR}}^{\mathrm{APR}}(3, n, \mathrm{IAC})$ and $P_{\mathrm{NMR}}^{\mathrm{APR}}(3, n, \mathrm{IAC})$ to $P^{\mathrm{APR}}(3, n, \mathrm{IAC})$, it seems clear that neither the NTR nor the NMR reduce the probability of the Strong Borda Paradox. When the electorate is infinite, the probability is 0,0486 both under the NTR and the NMR while it is 0,0295 when there is no restriction.

Table 9: Limiting probabilities of the Strong Borda Paradox

\begin{tabular}{|c|c|c|c|c|c|c|c|c|c|c|c|}
\hline & \multicolumn{11}{|c|}{$\lambda$} \\
\hline & $\mathbf{0}$ & 0.1 & 0.2 & 0.3 & 0.4 & 0.5 & 0.6 & 0.7 & 0.8 & 0.9 & 1 \\
\hline$\overline{P_{\mathrm{NBR}}(3, \lambda, \infty)^{*}}$ & 0.0278 & 0.0176 & 0.0094 & 0.0036 & 0.0006 & 0 & 0 & 0 & 0 & 0 & 0 \\
\hline$P_{\text {NMR }}(3, \lambda, \infty)$ & 0.1111 & 0.0809 & 0.0525 & 0.0273 & 0.0081 & 0 & 0.0049 & 0.0152 & 0.0268 & 0.0383 & 0.0486 \\
\hline$P_{\mathrm{NTR}}(3, \lambda, \infty)$ & 0 & 0 & 0 & 0 & 0 & 0 & 0.0014 & 0.0078 & 0.0187 & 0.0328 & 0.0486 \\
\hline
\end{tabular}

* From Lepelley et al. (2000b).

Table 9 reports the limiting probabilities (when $n \rightarrow \infty$ ) of the strong Borda paradox given $\lambda \in[0,1]$ and given the restriction. We notice what follows: under the NBR, the Plurality rule $(\lambda=0)$ exhibits the highest limiting probability of the Strong Borda Paradox and that this probability tends to decrease as $\lambda$ grows. The Plurality rule $(\lambda=0)$ also exhibits the highest limiting probability under the NMR; under this restriction, the limiting probability tends to decrease with $\lambda \in\left[\begin{array}{ll}0 & \frac{1}{2}[\text {, }\end{array}\right.$ 
then it increases with $\left.\lambda \in] \frac{1}{2} 1\right]$. Under the NTR, we notice that the limit probability tends to increase with $\left.\lambda \in] \frac{1}{2} 1\right]$ and the Antiplurality rule $(\lambda=1)$ exhibits the highest limiting probability of the Strong Borda Paradox.

\section{Conclusion}

The aim of this paper was to find out under three well-known preference restrictions (NBR, NMR and NTR), the range of all the scoring rules that exhibit the Strong Borda Paradox. In our analysis, we proceeded by an analytical approach in order to reach the same results obtained in the literature by probability computations. In the social choice literature, Gehrlein and Lepelley (2010b,a) have provided for the Plurality, Antiplurality and the Borda rules, formulas of the Strong Borda Paradox's likelihood given each of the restrictions. These formulas are somewhat hard to handle. In this paper, we have provided a more attractive formula using the Barvinok's algorithm. Our probabilities under the NBR and the NTR are in line with those of Lepelley (1993), Lepelley et al. (2000b), Gehrlein and Lepelley (2010b,a). It came from our results that among the scoring rules, the Plurality rule tends to be more sensitive to the Strong Borda Paradox when preferences are restricted consistently the NBR or the NMR restriction; under the NTR restriction, it is the Antiplurality rule. It could be interesting to look at the probability representation or a formula that gives the limiting probabilities under the NMR given $\lambda$ as it has been done for the NBR by Lepelley et al. (2000b) and for the NTR by Gehrlein and Lepelley (2010b,a). It would also be interesting to extend the framework to more than three candidates as it has be done in Lepelley (1996) for the NBR.

Notice that one can easily generalize the NBR and the NTR restrictions to voting situations with more than three candidates; this is also the case for the NMR restriction when the number of candidates is odd. With an even number of candidates, the generalization of the NMR restriction will require a redefinition of what is the "middle". In such a case, several scenarios are possible and may open the path to further research. 


\section{Appendix: Simplified representations for the likeli- hood of the Strong Borda Paradox}

Formula 1. In a three-candidate election with single-peaked preferences, the likelihood of the Strong Borda Paradox for the Plurality rule is given by

$$
P_{N B R}^{P R}(3, n, I A C)=\left\{\begin{array}{ll}
\frac{n^{3}-6 n^{2}+32}{36 n(n+1)(n+5)} & \text { for } n \equiv 0[6] \\
\frac{n^{2}+2 n-35}{36 n(n+5)} & \text { for } n \equiv 1[6] \\
\frac{n(n-6)}{36(n+1)(n+5)} & \text { for } n \equiv 2[6] \\
\frac{(n-1)^{2}}{36 n(n+1)} & \text { for } n \equiv 3[6] \\
\frac{n^{3}-6 n^{2}-24 n+64}{36 n(n+1)(n+5)} & \text { for } n \equiv 4[6] \\
\frac{n^{3}+3 n^{2}-9 n-27}{36 n(n+1)(n+5)} & \text { for } n \equiv 5[6] \\
\frac{1}{36} & \text { for } n \rightarrow \infty
\end{array} \quad \text { for } n \geq 7\right.
$$

Formula 2. The likelihood of the Strong Borda Paradox for the Antiplurality rule in three-candidate elections with preferences satisfying the NTR restriction is given by

$$
P_{N T R}^{A P R}(3, n, I A C)=\left\{\begin{array}{ll}
\frac{7 n^{3}-12 n^{2}-144 n+864}{144 n(n+1)(n+5)} & \text { for } n \equiv 0[12] \\
\frac{n 7 n^{3}+15^{2}-51 n+29}{144 n(n+1)(n+5)} & \text { for } n \equiv 1[12] \\
\frac{7 n^{3}-12 n^{2}-252 n+496}{144 n(n+1)(n+5)} & \text { for } n \equiv 2[12] \\
\frac{7 n^{3}+15^{2}-63 n-135}{144 n(n+1)(n+5)} & \text { for } n \equiv 3[12] \\
\frac{7 n^{3}-12 n^{2}-240 n+704}{144 n(n+1)(n+5)} & \text { for } n \equiv 4[12] \\
\frac{7 n^{3}+15^{2}+45 n+253}{144 n(n+1)(n+5)} & \text { for } n \equiv 5[12] \\
\frac{7 n^{3}-12 n^{2}-252 n+432}{144 n(n+1)(n+5)} & \text { for } n \equiv 6[12] \\
\frac{7 n^{2}-20 n-59}{144 n(n+1)} & \text { for } n \equiv 7[12] \\
\frac{7 n^{3}-12 n^{2}-144 n+928}{144 n(n+1)(n+5)} & \text { for } n \equiv 8[12] \\
\frac{7 n^{3}+15^{2}+45 n+189}{144 n(n+1)(n+5)} & \text { for } n \equiv 9[12] \\
\frac{7 n^{3}-12 n^{2}-348 n+272}{144 n(n+1)(n+5)} & \text { for } n \equiv 10[12] \\
\frac{7 n^{2}+8 n-71}{144 n(n+5)} & \text { for } n \equiv 11[12] \\
\frac{7}{144} & \text { for } n \rightarrow \infty
\end{array} \quad \text { for } n\right.
$$


Formula 3. The likelihood of the Strong Borda Paradox for the Plurality rule in three-candidate elections with preferences satisfying the NMR restriction is given by

$$
P_{N M R}^{P R}(3, n, I A C)=\left\{\begin{array}{ll}
\frac{4 n^{3}-33 n^{2}+18 n+216}{36(n+1)(n+5)} & \text { for } n \equiv 0[6] \\
\frac{2 n^{3}-3 n^{2}-18 n+19}{18 n(n+1)(n+5)} & \text { for } n \equiv 1[6] \\
\frac{4 n^{3}-33 n^{2}-6 n+112}{36 n(n+1)(n+5)} & \text { for } n \equiv 2[6] \\
\frac{2 n^{3}-3 n^{2}-18 n+27}{18 n(n+1)(n+5)} & \text { for } n \equiv 3[6] \\
\frac{4 n^{3}-33 n^{2}+18 n+200}{36 n(n+1)(n+5)} & \text { for } n \equiv 4[6] \\
\frac{2 n^{2}-5 n-25}{18 n(n+5)} & \text { for } n \equiv 5[6] \\
\frac{1}{9} & \text { for } n \rightarrow \infty
\end{array} \quad \text { for } n \geq 7\right.
$$

Formula 4. Given $n$ the number of voters, the likelihood of the Strong Borda Paradox for the Antiplurality rule in three-candidate elections with preferences satisfying the NMR restriction is given by

$$
P_{N M R}^{A P R}(3, n, I A C)=\left\{\begin{array}{ll}
\frac{7 n^{2}-48 n+144}{144(n+1)(n+5)} & \text { for } n \equiv 0[12] \\
\frac{7 n^{3}+15 n^{2}-51 n+29}{144 n(n+1)(n+5)} & \text { for } n \equiv 1[12] \\
\frac{7 n^{3}-48 n^{2}+36 n+64}{144 n(n+1)(n+5)} & \text { for } n \equiv 2[12] \\
\frac{7 n^{3}+15 n^{2}-63 n-135}{144 n(n+1)(n+5)} & \text { for } n \equiv 3[12] \\
\frac{7 n^{3}-48 n^{2}+48 n+128}{144 n(n+1)(n+5)} & \text { for } n \equiv 4[12] \\
\frac{7 n^{3}+15 n^{2}+45 n+253}{144 n(n+1)(n+5)} & \text { for } n \equiv 5[12] \\
\frac{7 n^{2}-48 n+36}{144(n+1)(n+5)} & \text { for } n \equiv 6[12] \\
\frac{7 n^{2}-20 n-59}{144 n(n+1)} & \text { for } n \equiv 7[12] \\
\frac{7 n^{3}-48 n^{2}+144 n+64}{144 n(n+1)(n+5)} & \text { for } n \equiv 8[12] \\
\frac{7 n^{3}+15 n^{2}+45 n+189}{144 n(n+1)(n+5)} & \text { for } n \equiv 9[12] \\
\frac{7 n^{3}-48 n^{2}-60 n+128}{144 n(n+1)(n+5)} & \text { for } n \equiv 10[12] \\
\frac{7 n^{2}+8 n-71}{144 n(n+5)} & \text { for } n \equiv 11[12] \\
\frac{7}{144} & \text { for } n \rightarrow \infty
\end{array} \quad \text { for } n\right.
$$




\section{References}

Barvinok A., 1994. Polynomial time algorithm for counting integral points in polyhedra when the dimension is fixed. Mathematics of Operations Research 19, 769-779.

Barvinok A., Pommersheim J., 1999. An algorithmic theory of lattice points in polyhedra. In: New Perspectives in Algebraic Combinatorics, Berkeley, CA, 1996-1997. Math. Sci. Res. Inst. Publ. 38, 91-147.

Berg S., Lepelley D., 1994. On probability models in voting theory. Statistica Neerlandica 48, 133-146.

Black D., 1958. The Theory of Committees and Elections. Cambridge University Press, Cambridge.

Bruynooghe M., Cools R., Verdoolaege S., Woods K. 2005. Computation and manipulation of enumerators of integer projections of parametric polytopes. Technical Report CW 392. Katholieke Universiteit Leuven, Department of Computer Sciences.

Clauss P., Loechner V., 1998. Parametric analysis of polyhedral iteration spaces. Journal of VLSI Signal Processing 2(19), 179-194.

Diss M., Gehrlein W.V., 2012. Borda's paradox with weighted scoring rules. Social Choice and Welfare 38, 121-136.

Ehrhart E., 1962. Sur les polyèdres rationnels homothétiques à $n$ dimensions. Comptes Rendus de l'Academie des Sciences Paris 254, 616-618.

Ehrhart E., 1967. Sur un problème de géométrie diophantienne linéaire. Ph.D. Thesis. Journal für die Reine und Angewandte Mathematik. 226, 1-49.

Gehrlein W.V., Fishburn P.C., 1976. The probability of the paradox of voting: A computable solution. Journal of Economic Theory 13: 14-25.

Gehrlein WV., 2006. Condorcet's Paradoxes. Springer Publishing, Berlin.

Gehrlein WV., 2004. Consistency in measures of social homogeneity : A connection with proximity to single peaked preferences. Quality and Quantity 38: 147-171.

Gehrlein WV., 2002. Obtaining representations for probabilities of voting outcomes with effectively unlimited precision integer arithmetic. Social Choice and Welfare 19: 503-512. 
Gehrlein W.V., Lepelley D., 2010a. Voting Paradoxes and Group Coherence. Springer.

Gehrlein WV., Lepelley D., 2010b. On the probability of observing Borda's Paradox. Social Choice and Welfare 35: 1-23.

Huang H.C, Chua V.C., 2000. Analytical representation of probabilities under IAC condition. Social Choice and Welfare 17: 143-155.

Lepelley D., 1996. Constant Scoring rules, Condorcet criteria and Single-peaked preferences. Economic Theory 7(3): 491-500.

Lepelley D., 1993. On the probability of electing the Condorcet loser. Mathematical social Sciences 25: 105-116.

Lepelley D., Louichi A., Smaoui H., 2008. On Ehrhart polynomials and probability calculations in voting theory. Social Choice and Welfare 30(3):363-383.

Lepelley D., Louichi A., Valognes F., 2000a. Computer simulations of voting systems. In Ballot $\mathrm{G}$ and Weisbuch $\mathrm{G}$ (eds). Applications of simulations to social sciences, Hermes, Oxford, 181-194.

Lepelley D., Pierron P., Valognes F., 2000b. Scoring rules, Condorcet efficiency and social homogeneity. Theory and Decision 49: 175-196.

Saari D., 1995. Basic Geometry of Voting. Berlin, Heidelberg, New York, Springer.

Saari D., Valognes F., 1999. The Geometry Of Black's Single Peakedness And Related Conditions. Journal of Math Econ 32:429-456.

Sen D., 1966. A possibility Theorem on Majority Decisions. Econometrica 34(2):491499.

Tataru M, Merlin V., 1997. On the relationship of the Condorcet winner and positional voting rules. Mathematical Social Sciences 34: 81-90.

Verdoolaege S., Seghir R., Beyls K., Loechner V., Bruynooghe M., 2004. Analytical computation of Ehrhart polynomials: enabling more compiler analysis and optimizations, in: Proceedings of International Conference on Compilers, Architecture and Synthesis for Embedded Systems, Washington DC.

Vickery W., 1960. Utility, strategy and social decision rules. Quaterly Journal of Economics 74:507-535. 
Ward B., 1965. Majority voting and alternative forms of public enterprises. In Margolis J. (eds): Public economy of urban communities. J. Hopkins press, Baltimore, pp 112-126.

Wilson M. C., Pritchard G., 2007. Probability calculations under the IAC hypothesis. Mathematical Social Sciences 54:244-256. 